\title{
Comparison of the $\mathrm{x}$-ray spectroscopy response and charge transport properties of semi-insulating In/AI doped CdZnTe crystals
}

\author{
Y. Xu, ${ }^{1,2, a)}$ P. J. Sellin, ${ }^{2}$ A. Lohstroh, ${ }^{2}$ W. Jie, ${ }^{1}$ T. Wang, ${ }^{1}$ C. Mills, ${ }^{2}$ P. Veeramani, ${ }^{2}$ \\ and M. Veale ${ }^{2}$ \\ ${ }^{1}$ School of Materials Science and Engineering, Northwestern Polytechnical University, Xi'an 710072, China \\ ${ }^{2}$ Department of Physics, University of Surrey, Guildford GU2 7XH, United Kingdom
}

(Received 15 October 2008; accepted 6 February 2009; published online 16 April 2009)

\begin{abstract}
The x-ray spectroscopy performance of $\mathrm{In} / \mathrm{Al}$ doped CdZnTe planar detectors based on as-grown crystals were investigated at room temperature, using a $\mathrm{Tb}$ x-ray source with a principle energy of $44.2 \mathrm{keV}$. The observed broadening in the photopeak resolution was attributed to incomplete charge carrier collection due to carrier trapping and scattering by the defects in the crystal. Alpha particle spectroscopy and pulse shape rise time analysis were used to measure the electron mobility lifetime product $(\mu \tau)$, as well as the mobility $(\mu)$ of the CdZnTe material grown with different dopant concentrations. To further clarify the role of the dopant and associated trapping states, temperature dependent alpha particle spectroscopy and pulse shapes were investigated at various applied bias fields over a temperature range from 200 to $300 \mathrm{~K}$. CdZnTe doped with $1.5 \mathrm{ppm}$ In exhibits excellent $\mathrm{x}$-ray spectral resolution and charge transport properties, which implies a lower density of trapping centers in the crystal. The deep levels associated with $\mathrm{Cd}_{i}^{2+}$ have been tentatively recognized as electron trapping centers and this is confirmed by the observed reduction in electron lifetime in CdZnTe crystal with $15 \mathrm{ppm}$ In. Additionally, a shallower electron detrapping defect, with an activation energy of $0.14 \pm 0.02 \mathrm{eV}$, was also discovered to be simultaneously present in the crystal. In 30 ppm Al doped CdZnTe, however, the carrier mobility was significantly degraded due to scattering of the ionized centers attributed to the aluminum interstitial $\mathrm{Al}_{i}$. (C) 2009 American Institute of Physics. [DOI: 10.1063/1.3097301]
\end{abstract}

\section{INTRODUCTION}

Interest in the use of cadmium zinc telluride $(\mathrm{CdZnTe})$ based nuclear radiation detectors for medical imaging and security applications has grown rapidly in recent years. This is driven by the potential of CdZnTe to provide excellent energy resolution for hard $\mathrm{x}$-ray and gamma ray spectroscopy at room temperature. ${ }^{1,2}$ In principle, the resolution of an ideal spectroscopy detector is determined by the statistical fluctuation of the electron-hole pairs (ehps) generated by $\mathrm{x}$-ray or gamma ray photoelectric interaction in the bulk crystal, which is directly proportional to the energy of the incident photon. The excited ehps drifted through the bulk semiconductor toward the anode and cathode due to the influence of the electrical field. As they drifted, a transient charge is induced on the electrodes which formed a current pulse in the external circuit. The energy of the incident event is then recreated from the pulse amplitude and recorded in a pulse height spectrum using a multichannel analyzer (MCA).

Generally, in terms of the simple-element planar detector (with an infinitely large contact), the total measured variance of the observed photopeak, $\sigma_{\text {tot }}^{2}$, in the pulse height spectra is determined by the sum of three independent variances, ${ }^{3}$

$$
\sigma_{\text {tot }}^{2}=\sigma_{F}^{2}+\sigma_{e l}^{2}+\sigma_{c}^{2},
$$

where $\sigma_{F}^{2}$ is the variance of the noise due to carrier generation or Fano noise, $\sigma_{e l}^{2}$ is the variance of the noise owing to

\footnotetext{
a) Author to whom correspondence should be addressed. Electronic mail: npuxyd220@hotmail.com.
}

the leakage current and amplifier noise, and $\sigma_{c}^{2}$ is the variance of the noise owing to incomplete charge collection. In this paper, three as-grown, semi-insulating CdZnTe crystals doped by In/Al were selected and used to fabricate planar detectors with similar configurations. The spectroscopy response of the detectors to a $\mathrm{Tb} \mathrm{x}$-ray source with a principal energy of $44.2 \mathrm{keV}$ was presented and compared. Incomplete charge collection contributing to the resolution degradation is discussed. In practice, there are several mechanisms leading to incomplete charge collection, related to not only the CdZnTe crystal properties but also the specific device configuration. ${ }^{4,5}$ Typically, the latter can be ignored when considering planar detector. Therefore, in our measurements the observed variations in energy resolution between devices can be mostly attributed to the differences in the material characteristics.

As commonly known, the charge state and compensation efficiency of various dopants vary according to the precise nature of the dopants used and their compensation mechanism. Consequently, dopant behavior is recognized as a significant factor associated with charge carrier trapping or scattering. In this work, particular emphasis is given to the spectroscopic performance of the detectors, by varying the electrical field strength applied to the appropriate electrodes, which is simultaneously combined with the charge transport properties of the detectors. We attempt to explain the resulting trends in terms of the dopant behavior in order to find the optimal dopant compensation for use in the development of CdZnTe crystal growth technologies. 
TABLE I. Properties of the doped CdZnTe detectors. $(\mu \tau)_{e}$ and $\mu_{e}$ were measured at room temperature.

\begin{tabular}{lccccc}
\hline \hline Detector & $\begin{array}{c}\text { Thickness } \\
(\mathrm{mm})\end{array}$ & $\begin{array}{c}\text { Dopant level } \\
(\mathrm{ppm})\end{array}$ & $\begin{array}{c}\text { Resistivity } \\
(\Omega \mathrm{cm})\end{array}$ & $\begin{array}{c}(\mu \tau)_{e} \\
\left(\mathrm{~cm}^{2} / \mathrm{V}\right)\end{array}$ & $\begin{array}{c}\mu_{e} \\
\left(\mathrm{~cm}^{2} / \mathrm{V} \mathrm{s}\right)\end{array}$ \\
\hline CZT1 & 3.5 & $15 \mathrm{In}$ & $4.1 \times 10^{10}$ & $0.6 \times 10^{-3}$ & 640 \\
CZT2 & 2 & $1.5 \mathrm{In}$ & $3.4 \times 10^{10}$ & $2.3 \times 10^{-3}$ & 1010 \\
CZT3 & 1 & $30 \mathrm{Al}$ & $7.5 \times 10^{10}$ & $0.4 \times 10^{-3}$ & 175 \\
\hline \hline
\end{tabular}

\section{EXPERIMENTAL}

High resistivity $\mathrm{Cd}_{0.9} \mathrm{Zn}_{0.1}$ Te crystals (presented in Table I) were grown using the modified vertical Bridgman (MVB) method, which is described in more detail in a former paper. ${ }^{6}$ Three as-grown samples, named CZT1, CZT2, and CZT3, doped with $15 \mathrm{ppm}$ In, $1.5 \mathrm{ppm}$ In and $30 \mathrm{ppm} \mathrm{Al}$, respectively, were cut into the wafers with the thickness varying from 1 to $3.5 \mathrm{~mm}$, free from twins and grain boundaries. The samples were mechanically lapped and polished, and finally etched by $\mathrm{Br}$-methanol prior to making the detectors. Au contacts $(70 \mathrm{~nm}$ thick) were deposited onto both faces of the samples using thermal evaporation. Subsequently, passivation of the remaining free CdZnTe surface was performed to minimize surface leakage current using a $30 \% \mathrm{H}_{2} \mathrm{O}_{2}$ solution. Finally, one face of the completed detector was bonded by silver paste to a ceramic chip and the opposite electrode was connected using a wire.

X-ray spectroscopy was carried at room temperature when irradiated by an uncollimated terbium $(\mathrm{Tb})$ fluorescence x-ray source with the $K_{\alpha}$ and $K_{\beta}$ peak energies of 44.2 and $50.7 \mathrm{keV}$, respectively. During the measurements, the bias voltage was applied to the irradiated contact via a charge sensitive preamplifier (eV Products modal 550), from a lownoise bias supply (ORTEC 710). The preamplifier output signals were shaped by a spectroscopy amplifier (ORTEC 570) with an optimized shaping time of $2 \mu \mathrm{s}$, followed by acquisition of pulse height spectra using a standard MCA (CANBERRA Multiport).

The charge transport properties of the CdZnTe material were evaluated using alpha particle spectroscopy. The measurements were carried out by placing the devices inside a temperature-controlled vacuum chamber at pressure below $2 \times 10^{-2} \mathrm{~Pa}$, with the sample temperature varied in the range from 200 to $300 \mathrm{~K}$. The pulse height spectra and the rise time distribution from the CdZnTe detectors were induced by an uncollimated ${ }^{241} \mathrm{Am} 5.48 \mathrm{MeV}$ alpha particle source. Pulse shape information was simultaneously recorded by connecting the preamplifier output to a high-speed waveform digitizer card. The CdZnTe detectors were electrically characterized by current-voltage $(I-V)$ measurements using a picoammeter/voltage supply (Keithley 487).

\section{RESULTS AND DISCUSSION}

\section{A. X-ray spectroscopy}

The measured $\mathrm{Tb}$ x-ray response spectra for CZT1, CZT2, and CZT3 at various bias voltages (electrical field strength) are demonstrated in Figs. 1(a)-1(c), respectively. Generally, the full width at half maximum (FWHM) of the full-energy peaks was assumed to be a measure of the energy resolution of the detector. The position of photopeak centroid is dependent on the charge collection efficiency (CCE) and so is strongly bias dependent. The spectroscopy response of the three detectors to the $\mathrm{Tb}$ source is analyzed by evaluating the FWHM and photopeak centroid, as illustrated in Figs. $1(\mathrm{~d})-1(\mathrm{f})$.

For CZT2, Fig. 1(e) shows a decreasing photopeak FWHM with increasing applied electrical field and a slight improvement in centroid to higher channel numbers. This implies that the detector is close to saturation of the sensitivity and is able to achieve a CCE approximate to 1.0. Besides, the good charge transport performance of this device is confirmed by the $K_{\beta}$ peak, which is weakly resolved as a high energy shoulder in the spectrum. These data confirm the good crystalline quality of the CdZnTe material and the absence of significant concentrations of electron trapping centers. This is in good agreement with the higher electron mobility lifetime product $\left(\mu \tau_{e}=2.3 \times 10^{-3} \mathrm{~cm}^{2} / \mathrm{V}\right)$ by alpha particle measurements at room temperature, as illustrated in Fig. 2 , and the higher electron mobility $\left(\mu_{e}\right.$ $=1010 \mathrm{~cm}^{2} / \mathrm{V} \mathrm{s}$ ) by the shorter transient time of the electrons reflected in the alpha pulse shape, as illustrated in Fig. 3.

In the case of CZT1, there is a more rapid improvement of the FWHM when increasing the bias, however, the FWHM is still wide as compared with CZT2. A significant shift of the photopeak centroid without saturation, over a wide range of the electrical field, can be observed in Fig. 1(d), indicating a steady increase in the mean electron drift length at higher field strength. This is consistent with the generally lower electron mobility lifetime and drift mobility values observed in this sample from alpha particle measurements, as listed in Table I. Deep-level trapping centers, related to $\mathrm{Cd}_{i}^{2+}$, likely to account for the electron trapping, were discussed in our previous study. ${ }^{8}$ Some of the ephs were caught by the deep level traps and causes the incomplete charge collection. Additionally, $\mathrm{Cd}_{i}^{2+}$ species are known to degrade the performance of CdTe radiation detectors, acting as mobile centers that can easily diffuse toward the cathode and strongly affect the local electric field distribution in the device. ${ }^{9}$

The pulse shapes from CZT1 shows a significant slow component of the induced charge pulse, in addition to the prompt drift part of the pulse shape, as illustrated in Fig. 3. This slow transient is due to thermal emission of charge from a shallower trap existing in the material. Since the shallow traps allow detrapping of charge, they may not limit the overall amplitude of the pulse provided that the charge is reemitted sufficiently fast compared to the shaping time of the spectroscopy amplifier. Similar phenomena have been reported from thermally emitted charge transients in high purity synthetic diamond detectors. ${ }^{10}$ The $10 \%-60 \%$ rise time $\tau_{10 \%-60 \%}$ distribution of the pulses under various bias voltages were acquired and used to calculate the electron mobility shown in Table I.

Contrary to CZT3, the photopeak centroid gradually becomes saturated at higher electrical field [Fig. 1(f)], with a relatively low CCE compared to CZT2. This crystal is doped 

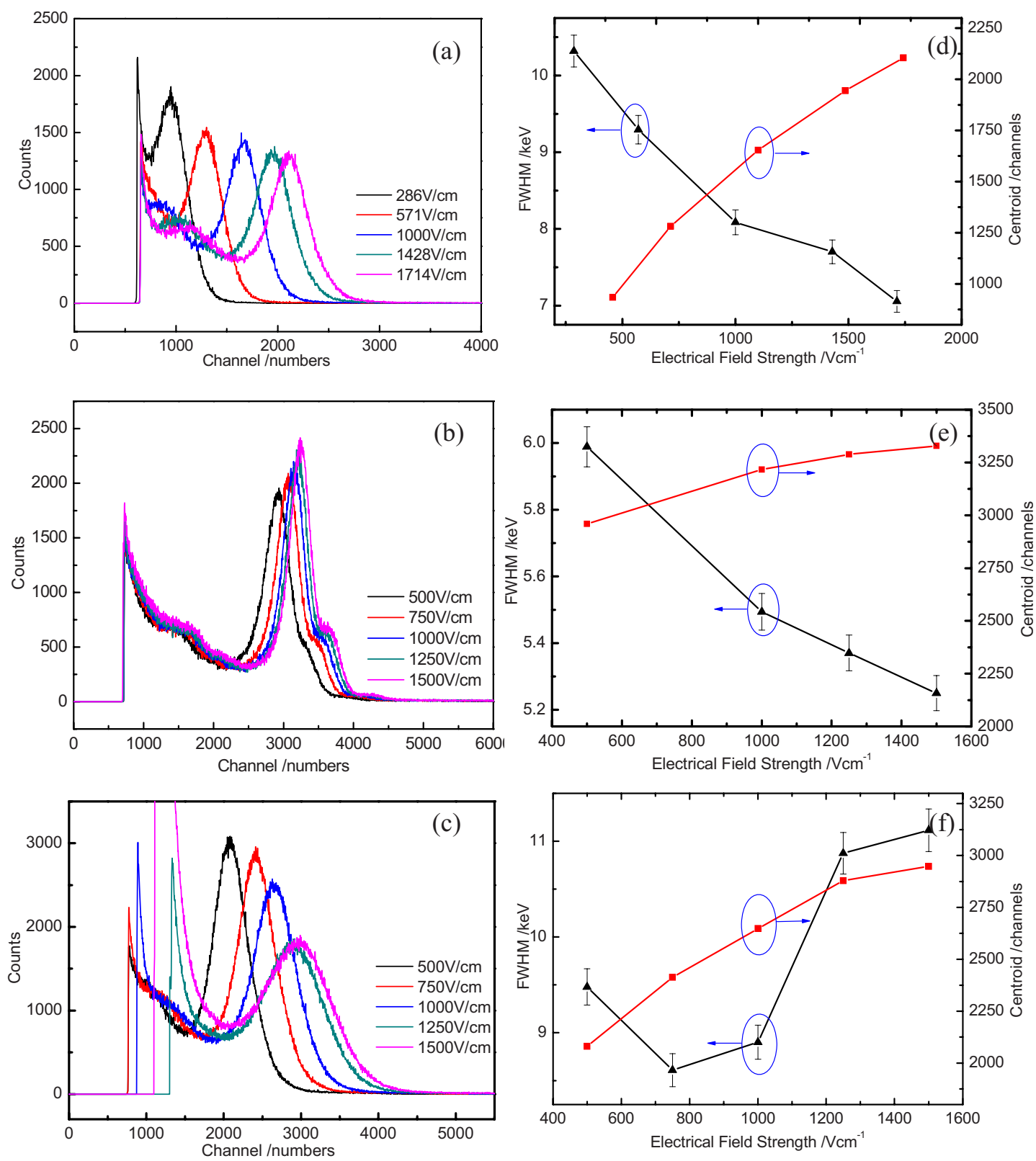

FIG. 1. (Color online) Comparison of the x-ray response of (a) CZT1, (b) CZT2, and (c) CZT3, respectively, irradiated by an uncollimated Tb x-ray source with $K_{\alpha}$ and $K_{\beta}$ peak energies of 44.2 and $50.7 \mathrm{keV}$ at various bias voltages. The centriod and FWHM values of the peaks are plotted for each of the detectors in (d)-(f), respectively.

with $\mathrm{Al}$ with a higher concentration of $30 \mathrm{ppm}$, which could produce an average high concentration of aluminum interstitials $\mathrm{Al}_{i}$. The presence of ionized interstitials throughout the bulk of the crystal may tend act as scattering centers which will significantly degrade the electron mobility. Both low electron mobility-lifetime product $\left(\mu \tau_{e}=4 \times 10^{-4} \mathrm{~cm}^{2} / \mathrm{V}\right)$ and low drift mobility $\left(\mu_{e}=175 \mathrm{~cm}^{2} / \mathrm{V} \mathrm{s}\right)$ are observed in this sample, as shown in Table I. Figure 3 also shows that the induced alpha pulse for CZT3 has a significantly slower rise time than CZT1 and CZT2, despite the thinner sample.

On the other hand, it is evident from Fig. 1(c) that the tail effect is very severe, particularly at high electrical field, which is possibly exacerbated by the large leakage current observed in this device at negative bias, shown in Fig. 4. This effect can be seen by the development of the FWHM as the voltage is increased, as seen in Fig. 1(f). Initially the FWHM decreases as the electric field strength increases to about $800 \mathrm{~V} \mathrm{~cm}^{-1}$, consistent with increased drift velocity and improved charge transport. However, at field strengths above $800 \mathrm{~V} \mathrm{~cm}^{-1}$, the increased leakage current produced causes the electronic noise ( $\sigma_{e l}^{2}$ variance) to dominate, which broadens the photopeak.

In addition, if taken into account, several other material properties would tend to reduce CCE and deteriorate the energy resolution of the detectors. Two leading causes among these effects are dislocations and precipitates. It is likely that the high density of dislocations acts as recombination centers and therefore produces local variation in the carrier lifetime. ${ }^{11}$ It is expected that the presence of precipitates in a crystal, such as Te inclusions/precipitates in CdZnTe, affects both the variance in charge pair creation and the variance in charge transport by trapping the charge carriers and distorting the local electric field. ${ }^{12}$ Nevertheless, such short-range local fluctuations can become of critical importance for 


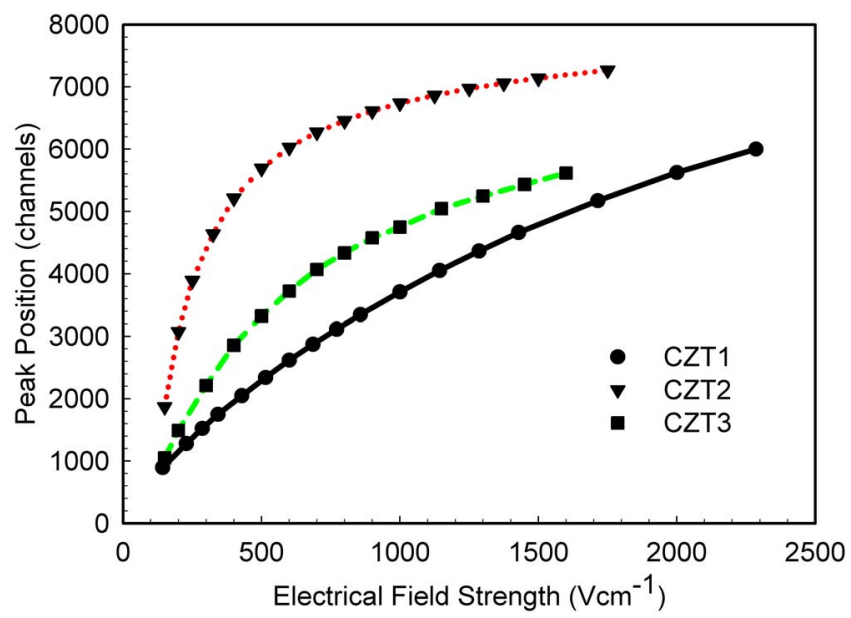

FIG. 2. (Color online) Comparison of the electron mobility lifetime products fitted using the single carrier Hecht equation (Ref. 7). The $(\mu \tau)_{e}$ are $0.6 \times 10^{-3}, 2.3 \times 10^{-3}$, and $0.4 \times 10^{-3} \mathrm{~cm}^{2} / \mathrm{V}$ for CZT1, CZT2, and CZT3, respectively.

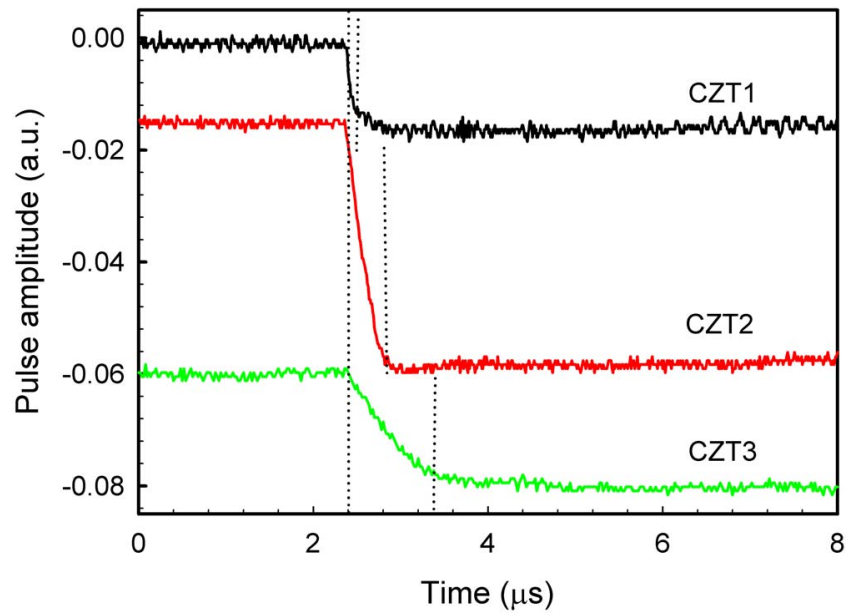

FIG. 3. (Color online) Temporal measurements at room temperature under an applied electric field strength of $600 \mathrm{~V} / \mathrm{cm}$ for CZT1, CZT2, and CZT3. The region between the dashed lines is selected for the rise time calculation. Measurements at $300 \mathrm{~V} / \mathrm{cm}$ and above $1000 \mathrm{~V} / \mathrm{cm}$ showed similar properties.

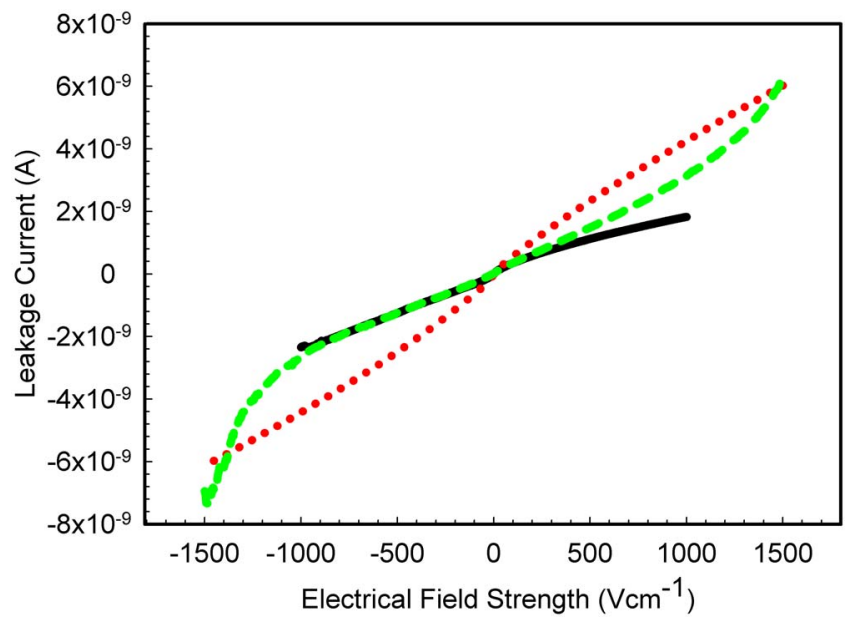

FIG. 4. (Color online) Typical $I-V$ curves of the Au/CdZnTe/Au detectors made of CZT1 (solid line), CZT2 (dotted line), and CZT3 (dashed line).
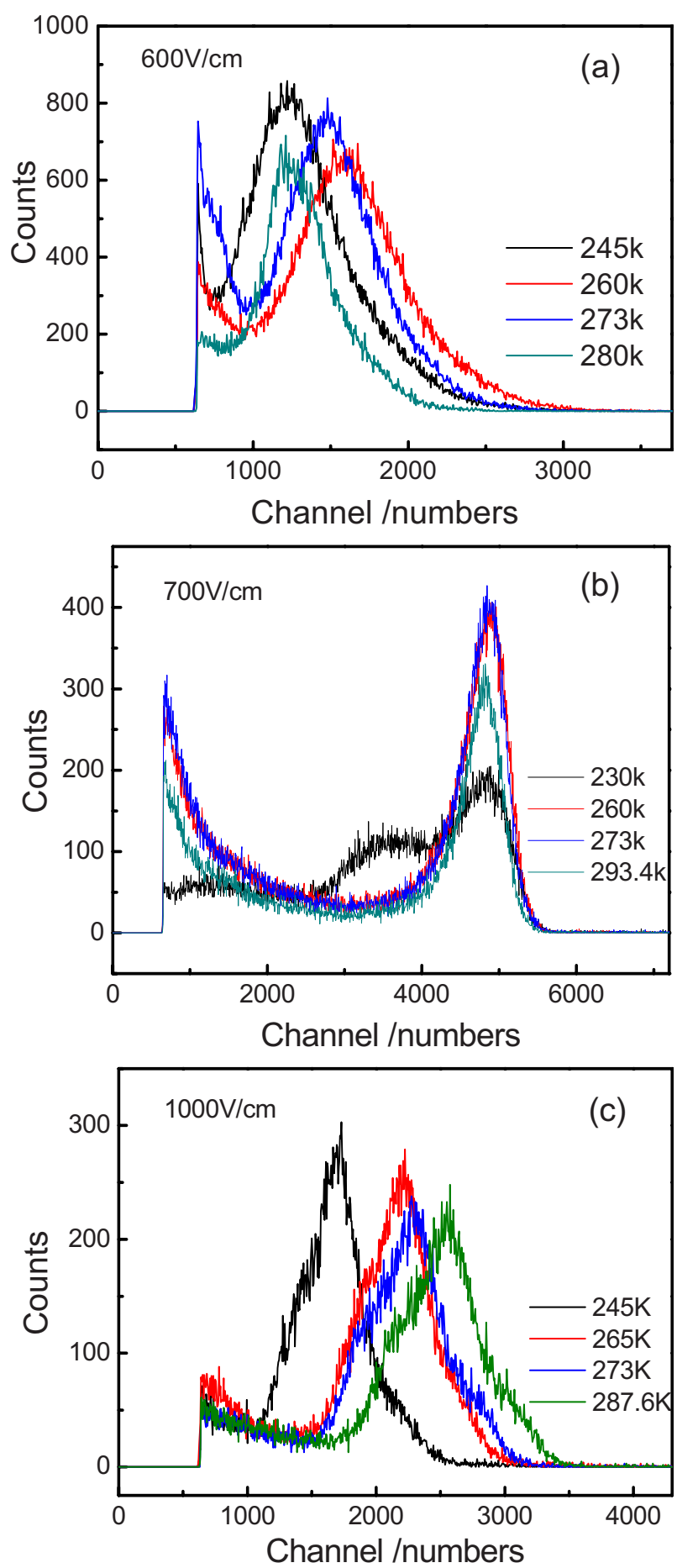

FIG. 5. (Color online) Temperature dependent $\alpha$-particle spectra for (a) CZT1, (b) CZT2, and (c) CZT3 measured at different applied electric field strengths.

highly pixellated imaging detectors, where pixel-to-pixel signal uniformity is critical. Moreover, the ratio of x-ray interaction depth with the thickness of the detector is also a potential issue to influence the $\mathrm{CCE}$, with respect to the different thicknesses of the detectors, and in particular for the thinnest one. ${ }^{13}$

\section{B. Temperature dependent $\alpha$-particle spectroscopy and pulse shape analysis}

To further clarify the role of the trapping or scattering centers in the crystals, the alpha particle spectroscopy and 

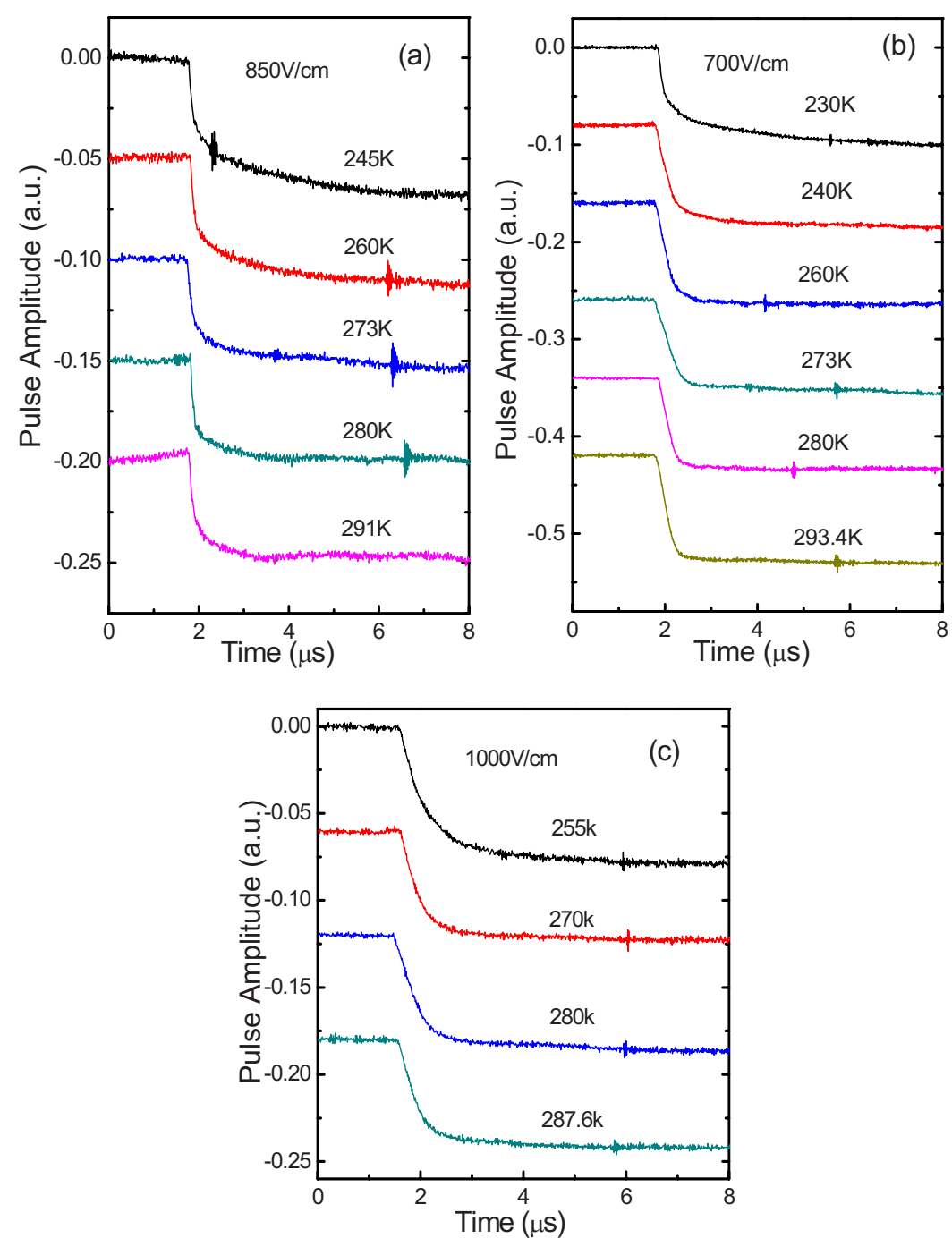

pulse shapes of the three detectors were investigated under various applied bias voltages over a temperature range from 200 to $300 \mathrm{~K}$. Figure 5 shows the typical alpha particle collection spectra of CZT1, CZT2, and CZT3, respectively, acquired at different temperatures. The photopeak centroid is proportional to the CCE and affected by the charge transport properties of the detector. The centroid position for CZT2 is almost unaffected as the temperature is lowered, implying that the mean electron drift length in the device is insensitive to temperature. This is consistent with a low concentration of trapping centers and high crystallographic quality of this sample. Simultaneously, the induced alpha pulse shapes were acquired and a selection of typical pulses are shown in Fig. 6(b). The transient time for the electron carrier is relatively fast, over a large range of temperatures, suggesting that the electron mobility is high and only slightly affected by changes in temperature. A significant slow component in the pulse shape seems to emerge up to $230 \mathrm{~K}$, which coincides with the broader pulse height spectrum and lower-energy satellite peak shown in Fig. 5(b).

For CZT1, the alpha peak centroid initially increases as the temperature is reduced to $260 \mathrm{~K}$, then decreases as the temperature is further reduced, as shown in Fig. 5(a). As the vibration and diffusion of the dominating deep level defects $\mathrm{Cd}_{i}^{2+}$ were weakened with decreasing temperature, the trap- ping is reduced for the irradiated ehps, improving the CCE. However, another kind of defect could participate in the electron transport, which is possibly responsible for the reduced CCE at temperatures below $260 \mathrm{~K}$.

To be able to identify this defect, the alpha pulse shapes as a function of temperature were evaluated, as illustrated in Fig. 6(a). The detrapping time $\left(\tau_{D}\right)$ distribution is directly measured from the exponential decay of the slow transient wave forms of the charge pulses described by Lohstroh et $a l .{ }^{14}$ The relationship between $\tau_{D}$ and the trap activation energy $E_{A}$ is given by ${ }^{15}$

$$
\tau_{D}=\frac{1}{c T^{2}} \exp \left(\frac{E_{A}}{k_{B} T}\right),
$$

where $c$ is a trapping specific constant, $k_{B}$ is the Boltzmann constant, and $T$ is the temperature. An Arrhenius plot of $\ln \left(\tau_{D} T^{2}\right)$ versus $1 / k_{B} T$, shown in Fig. 7 , gives a detrapping energy $E_{A}$ of $0.14 \pm 0.02 \mathrm{eV}$. This data provide direct evidence of one shallow level defect that potentially plays an important role in charge collection when the temperature is below $260 \mathrm{~K}$. This level defect is seemingly related to the $A$-center, an acceptorlike complex $\left[\operatorname{In}_{\mathrm{Cd}}^{+} \mathrm{V}_{\mathrm{Cd}}^{2-}\right]^{-}$formed by a shallow donor $\mathrm{In}_{\mathrm{Cd}}$ and $\mathrm{Cd}$ vacancy, ${ }^{16}$ which introduces an energy band gap less than $0.15 \mathrm{eV}$. 


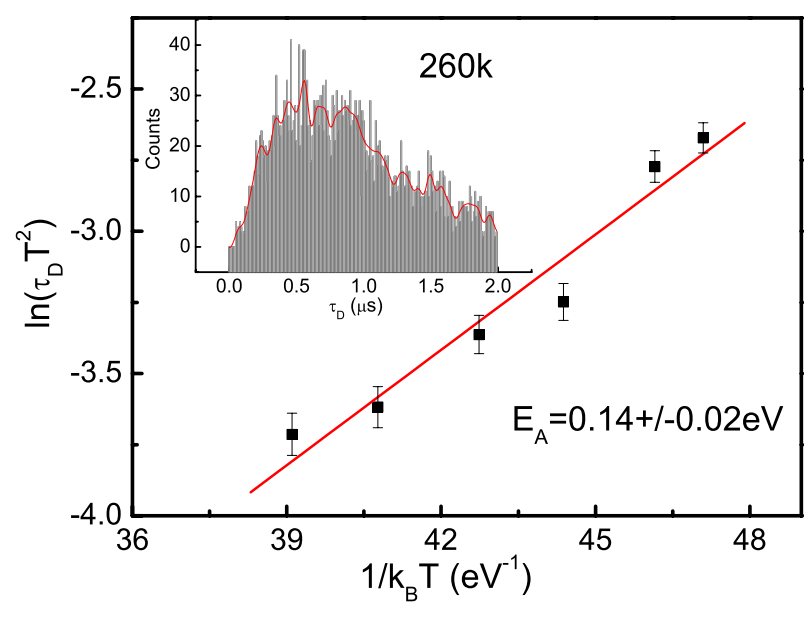

FIG. 7. (Color online) Arrhenius plot of $\ln \left(\tau_{D} T^{2}\right)$ as a function of $1 / k_{B} T$ for CZT1. The solid line is the result of fitting Eq. (2). The inset is a histogram of the detrapping time distribution at $260 \mathrm{~K}$.

For the heavy Al doped CZT3 shown in Fig. 5(c), the peak centroid dramatically shifts to lower channel number as the temperature is reduced, demonstrating a strong decrease in the CCE with decreasing temperature. This could be due to either a strongly reduced drift mobility at lower temperature, as observed in a trap-assisted conduction mechanism, or due to a highly reduced electric field distribution due to trapped space charge. In either mechanism, the reduced charge transport at low temperature is caused by a high trap concentration, which could be $\mathrm{Al}$ interstitials in the material. This nonideal behavior is confirmed by the pulse shapes, confined to a narrow temperature range, shown in Fig. 6(c). In this case at temperatures below $255 \mathrm{~K}$ the pulse shape loses the prompt drift component and the pulse amplitude becomes too small to be resolved.

\section{CONCLUSIONS}

The x-ray spectroscopy response of three CdZnTe planar detectors, fabricated on as-grown semi-insulating CdZnTe crystals and doped with different levels of $\mathrm{In} / \mathrm{Al}$, was investigated using an uncollimated $44.2 \mathrm{keV} \mathrm{Tb} \mathrm{x}$-ray source. The energy resolution of the detectors was evaluated by comparing the pulse height spectra at various bias voltages. The 1.5 ppm In doped CdZnTe exhibits good x-ray spectroscopy resolution duo to a higher mean electron drift length, caused by better charge transport properties. The $(\mu \tau)_{e}$ and $\mu_{e}$ did not change significantly within the temperature range of 200$300 \mathrm{~K}$, implying a lower density of electron trapping centers in the crystal. This is therefore considered as an optimal concentration for In doped CdZnTe grown by the MVB method.

The degradation of $\mathrm{x}$-ray spectroscopy response for 15 ppm In doped CdZnTe crystal is possibly related to the dominating deep level electron trapping centers of $\mathrm{Cd}_{i}^{2+}$, which results in a shorter charge carrier lifetime and consequently a reduced CCE. A shallow electron detrapping defect, with an activation energy of $0.14 \pm 0.02 \mathrm{eV}$, was identified to be simultaneously present in the crystal, and were attributed to the slow component of the induced alpha pulses by electron re-emission. The heavily $\mathrm{Al}$ doped ( $30 \mathrm{ppm}) \mathrm{CdZnTe}$ sample displayed a noticeably higher concentration of scattering centers causing a worse charge transport property, including a significantly reduced electron drift mobility that adversely affected the detector performance.

\section{ACKNOWLEDGMENTS}

This project has been supported by the National Natural Science Foundation of China under Grant No. 50336040. The author (Y.X.) would like to acknowledge the China Scholarship Council for supplying an award under the State Scholarship Fund to enable this study at the University of Surrey, UK.

${ }^{1}$ T. E. Schlesinger, J. E. Toney, H. Yoon, E. Y. Lee, B. A. Brunett, L. Franks, and R. B. James, Mater. Sci. Eng., R. 32, 103 (2001).

${ }^{2}$ C. Szeles, Phys. Status Solidi B 241, 783 (2004).

${ }^{3}$ A. Owens and A. Peacock, Nucl. Instrum. Methods Phys. Res. A 531, 18 (2004).

${ }^{4}$ A. G. Kozorezov, J. K. Wigmore, A. Owens, R. den Hartog, A. Peacock, and A. Hala, Nucl. Instrum. Methods Phys. Res. A 546, 209 (2005).

${ }^{5}$ H. H. Barrett, J. D. Eskin, and H. B. Barber, Phys. Rev. Lett. 75, 156 (1995).

${ }^{6} \mathrm{G}$. Li, X. Zhang, H. Hua, and W. Jie, J. Electron. Mater. 34, 1215 (2005).

${ }^{7}$ A. Ruzin and Y. Nemirovsky, J. Appl. Phys. 82, 4166 (1997).

${ }^{8}$ Y. Xu, W. Jie, P. Sellin, T. Wang, W. Liu, G. Zha, and P. Veeramani, J. Phys. D 42, 03505 (2009).

${ }^{9}$ M. Du, H. Takenaka, and D. J. Singh, Phys. Rev. B 77, 094122 (2008).

${ }^{10}$ M. Marinelli, E. Milani, A. Paoletti, A. Tucciarone, and G. Verona-Rinati, Phys. Rev. B 64, 195205 (2001).

${ }^{11}$ L. S. Pan, D. R. Kania, P. Pianetta, J. W. Ager III, M. I. Landstrass, and S. Han, J. Appl. Phys. 73, 2888 (1993).

${ }^{12}$ A. E. Bolotnikov, G. S. Camarda, G. A. Carini, Y. Cui, L. Li, and R. B. James, Nucl. Instrum. Methods Phys. Res. A 571, 687 (2007).

${ }^{13}$ T. Takahashi and S. Watanabe, IEEE Trans. Nucl. Sci. 48, 950 (2001).

${ }^{14}$ A. Lohstroh, P. J. Sellin, S. G. Wang, A. W. Davies, and J. M. Parkin, J. Appl. Phys. 101, 063711 (2007).

${ }^{15}$ D. K. Schroder, Semiconductor Material and Device Characterisation (Wiley, New York, 1998).

${ }^{16}$ W. Stadler, D. M. Hoffmann, H. C. Alt, T. Muschik, B. K. Meyer, E. Weigel, G. Müller-Vogt, M. Salk, E. Rupp, and K. W. Benz, Phys. Rev. B 51, 10619 (1995). 